\title{
Transphobic victimisation and perceptions of future risk: A large-scale study of the experiences of trans people in the UK
}

Sonja J Ellis, Louis Bailey and Jay McNeil

\begin{abstract}
Due to perceived non-conformity to conventional constructions of gender, trans people may be subject to overt victimisation (e.g. physical or sexual violence; verbal abuse) and as a result of those experiences (actual or 'witnessed') may fear future victimisation. While some existing work reports levels of transphobic victimisation, there is a dearth of research on perceived risk; and more importantly, exploring group differences in actual victimisation and perceived risk. Drawing on survey responses from 660 trans people, the current study sets out to explore levels of victimisation, perceived risk of victimisation, and group differences (gender identity; stage of transition) in both these phenomena. Findings show that congruent with work on systematic oppression and minority stress, perceived risk of victimisation outstrips actual experiences. Almost no group differences were found on a basis of gender identity. Conversely, those currently undergoing a process (or part of a process) of gender reassignment or transition were significantly more likely to report having been victimised, and to perceive themselves at risk of future victimisation than those at any other stage of transition. That levels of perceived risk outstripped actual experiences of victimisation suggests that, in a culture that privileges cisgender experiences, isolated experiences of victimisation invoke a heightened sense of fear in members of the wider trans community. These findings suggest that there is a pressing need for dedicated support services for trans people; especially those who are going through a process of transitioning.
\end{abstract}

\section{Keywords}

Transphobia

Trans

Victimisation

Social inclusion

Gender prejudice 


\section{Transphobic victimisation and perceptions of future risk: A large-scale study of the experiences of trans people in the UK}

Transphobia commonly refers to hostility, harassment and discrimination against people who are - or who are perceived to be - gender variant. As defined in the literature, transphobia encompasses a wide range of behaviour including 'emotional disgust toward' (Hill and Willoughby, 2005, p. 533) or 'societal discrimination and stigma of' (Sugano, Nemoto, \& Operario, 2006, p. 217) individuals who do not conform to traditional societal norms of sex or gender. While transphobia may include covert (or subtle) forms of discrimination against trans people, the focus of this paper is on overt victimisation (physical violence, sexual violence, verbal abuse) directed towards people who are trans or who have a trans history.

In the UK the term 'Trans' is used to encompass the diverse range of people who find their personal experience of gender differs from the way in which gender is conventionally constructed within society. Whilst some trans people may have a binary gender identity (i.e. as men or women, irrespective of assigned sex), others may use alternative labels to define their gender (e.g. 'bigender', 'androgyne', 'polygender') or not define their gender at all (e.g. 'non-binary'). Trans people comprise a significant minority of the UK population - estimated to be around 300,000 (Reed, Rhodes, Schofield \& Wylie, 2009) and continuing to increase. However, a large proportion of this population are relatively invisible, particularly those who have transitioned and whose appearance may be indistinguishable from cis-gender ${ }^{1}$ men and women.

While there is a well-established field of psychological research on homophobia (prejudice against those who are, or are perceived to be, gay) transphobia is still a largely understudied area. In the main, prejudice and discrimination against trans people has tended to be subsumed within studies of prejudice affecting LGB people under the catch-all term LGBT (e.g. see McDermott, Roen, \& Scourfield, 2008; Meyer, 2012). Whilst these studies have often included trans people within their samples, they have not specifically explored transphobia. Instead, prejudice against LGB and T people has been studied homogenously under the umbrella of 'homophobia' (e.g. McDermott et al., 2008) or 'anti-queer' prejudice (e.g. Meyer, 2012); despite transphobic prejudice being about gender non-conformity rather

\footnotetext{
${ }^{1}$ People whose gender identity and expression that which is typically associated with the sex they were assigned at birth.
} 
than sexuality. While trans people are likely to experience distal stressors (e.g. violence; rejection) in much the same way as LGB people, they also experience additional challenges which potentially make their experiences of discrimination substantially different (see Testa et al., 2015, pp. 66-67). Because in western society gender is rigidly viewed as inevitably immutable and binary there is considerable potential for transphobia when someone's trans status or history is revealed who might otherwise have been assumed to be cisgender. Furthermore, social and/or physical transition may make some trans people more visible as gender variant and potentially at risk of transphobic prejudice. This is a distinctly trans experience. For all these reasons, to understand experiences of transphobia, it is both desirable and necessary to study transphobia in its own right rather than as a variation on, or extension of, homophobia.

A few select, and recent, studies have focused specifically on transphobia. In the main, these focus on attitudes towards trans people, in particular, among relatively large student samples (e.g. Hill \& Willoughby, 2005; Nagoshi et al., 2008; Tebbe \& Moradi, 2012). Lagging some 30 years behind the literature on homophobia, these studies have centred on the development, application, and standardisation of transphobia scales designed to measure attitudes towards trans people and gender non-conformity. While some studies (e.g. Nagoshi et al., 2008; Tebbe \& Moradi, 2012) have explored correlations between homophobia and factors such as age and gender; few consistent findings have emerged. Never-the-less, in one study (Nagoshi et al., 2008) transphobia was found to be highly correlated with socially conservative attitudes and adherence to conventional social norms around gender; a finding that is hardly surprising. However, a number of these studies report that response patterns tended not to conform to socially desirable norms, and transphobic views were not difficult to elicit (Hill \& Willoughby, 2005; Tebbe \& Moradi, 2012). This is very unusual in that most studies using standardised measures of prejudice have over several decades found a tendency for response patterns to conform to an anti-discrimination norm (e.g. see McConahay, Hardee \& Batts, 1981; Maison, 1995).

In addition to attitudinal studies, transphobia is often explored in relation to health and wellbeing as part of a correlational study (e.g. see Boza \& Nicholson Perry, 2014; Lombardi, 2009; Sugano et al., 2006). These studies indicate clear links between transphobic victimisation and poor outcomes in mental health and wellbeing; especially where an individual has had a greater number of transphobic victimisation experiences (e.g. Bariola et 
al., 2015). Congruent with this, recent research (e.g. Cramer et al., 2015) - albeit with an LGB sample - has found victimisation to be a mediating factor in internalised prejudice. While this in itself is important, these studies offer limited insight into the incidence and prevalence of transphobic victimisation.

Typically, acts of victimisation are perpetrated by members of the majority group against individuals who are (or are perceived to be) members of the minority group. As highlighted by Herek and colleagues (1999), victimisation is not simply an attack on an individual but rather a symbolic act intended to invoke fear into the collective group to which the individual belongs (or is perceived to belong). Transphobic victimisation is specifically (but not exclusively) linked to normative gender appearance/behaviour and is therefore designed to maintain the subordination of forms of gender presentation which do not conform to stereotypical social norms (cf. Bell \& Perry, 2015). As symbolic acts on the trans community as a whole, acts of victimisation invariably impact on perceptions of future risk. This form of oppression creates a 'climate of fear' (see Kitzinger, 1996) in which individuals see themselves as potential targets of victimisation. Feeling vulnerable may contribute to minority stress (Meyer, 1995) resulting in negative health outcomes such as internalised transphobia, anxiety, and low self-esteem.

Reported levels of transphobic victimisation appear to vary somewhat between studies. For example, in one study (Lombardi et al., 2002) 60\% of participants had experienced some form of harassment or violence, compared with $79 \%$ (Turner, Whittle \& Combs, 2009) and $87.4 \%$ (Couch et al., 2007) in other studies. Similarly, the incidence of rape and sexual assault is reported to range between $10 \%$ in some studies (e.g. Couch et al., 2007) and around $30 \%$ in other studies (e.g. Nemoto, Böedeker, \& Iwamoto, 2011). Some of these differences could potentially be explained by sampling. For example, some studies have researched highly specific sub-groups of the trans population, such as transwomen with a history of sex work (Nemoto et al., 2011) and transwomen of colour (Sugano et al., 2006) whereas others (e.g. Couch et al., 2007; Turner et al., 2009) have used larger and more diverse samples.

Although existing work on transphobia provides useful insights into the experiences of people within the trans population, they are not necessarily representative of trans people more generally. In particular, existing studies do not give an indication of whether trans people's experiences differ as a result of key characteristics. Given that transphobia is heavily underpinned by gender conformity, it would be expected that the incidence of victimisation 
might differ on the basis of gender presentation (e.g. the extent to which a person's appearance is read by others unequivocally as 'male' or 'female') and the extent to which an individual is able to 'pass' as cis-gender. As a function of gender identity, some trans people's appearance may be visibly different from socially proscribed norms. Similarly, while transitioning, it would not be unusual for a trans person's appearance to perhaps be at odds with normative gendered perceptions. This therefore raises previously unexplored questions about whether those who identify as male or female (in a clear and constant way) are less likely to experience transphobic victimisation than those with a non-binary or fluid gender identity; and whether there are differences in the likelihood of experiencing transphobic victimisation for those at specific stages of the transition process. Building on the existing literature reviewed here, the purpose of this paper was to report on the actual (selfreported) experiences of transphobic victimisation and the self-perceived (future) risk of victimisation of a large, and diverse, self-selected sample of trans people in the UK. Given the paucity of research exploring group differences in experiences of transphobic victimisation, this study also set out to explore differences in levels of actual victimisation and self-perceived risk of victimisation as a function of both gender identity and stage of transition.

\section{Method}

The data presented here forms part of a larger study on mental health and wellbeing in the UK trans population (see McNeil, Bailey, Ellis, Morton \& Regan, 2012). Because of the paucity of data on this topic - in particular, the absence of UK data - survey methods were employed in order to gather baseline data from as large a sample as possible. To ensure the potential for international comparison, the questions embedded within our survey were developed with reference to existing large-scale survey work on mental health and wellbeing in trans people such as the Canadian Trans Pulse Project (Bauer et al., 2009) and the Australasian Tranznation study (Couch et al., 2007). As a marginalised group who have often not been treated well by psychologists and health professionals, trans people can sometimes be especially cautious about the motives of researchers attempting to access them. In order to minimise the risk of alienating members of the trans population, we engaged extensively with key stakeholders (e.g. professional and lay representatives of the trans community) in the early stages of the study. This stakeholder group provided valuable critical feedback about 
issues around terminology (e.g. what categories to use for 'gender identity' and 'sexual orientation'; whether to use 'trans' or other variations), the appropriateness of the items within the survey (resulting in the removal of some proposed questions) and alsoareas that could be explored which were not initially included.

In its entirety, the survey comprised 89 pages and a total of 187 questions; with a completion time of 1-2 hours. As described above, it was compiled by the authors of this paper (some of whom work closely with trans people in a professional capacity) with reference to existing published work as well as feedback from our stakeholder group. The substantive content of the survey comprised questions on a wide range of issues including, among other things, perceptions of life satisfaction, current mental health status/behaviours, experiences of daily life as a trans person, experiences of using gender-related and mental health services, sources of social support. For the purposes of this paper, only questions relating to experiences of transphobic victimisation and self-perceived risk of victimisation are reported. This comprised questions asking about occurrences of various types of transphobic incidents, how recently these occurred and respondents' self-perceived risk of these happening to them in the future (i.e. how often have you been sexually assaulted because you are trans? When did this last happen to you? Do you worry about this happening in the future?). This set of questions comprised forced-choice categorical response options. For each item (e.g. being made fun of; being hit or beaten up; etc) the question 'how often have you...?' comprised five response options (never; once; twice; a few times; many times), 'when did this last happen to you?' eight response options (...in the last year; in the last five years; in the last 10 years; over 10 years ago), and 'do you worry about this happening in the future?' two response options (yes; no). These particular questions were inituially derived from the Transpulse survey (http://transpulseproject.ca/resources/trans-pulse-survey/) and modified slightly - in consultation with the stakeholder group - for use in a British context.

Prior to launching the survey, the study received formal ethical approval from the Faculty Research Ethics Committee at Sheffield Hallam University. Given that the trans population is hard to reach, with many choosing to keep their trans status (or history) private, we opted to administer the survey electronically. While this undoubtedly meant that we didn't reach some members of the trans population, this method gave us the best chance of reaching people not engaged in support groups or other face-to-face fora. Currently there is no definitive way of identifying the trans population and therefore no identifiable base from which to draw a representative sample. Consequently, our recruitment relied heavily on self-selection elicited 
through email networks and supported by promotion by the research team at support groups and trans gatherings.

A briefing about the study and a direct link to the survey was disseminated through more than 70 organisations/groups in the UK. This included trans or LGBT organisations and networks based nationally (e.g. The National LGB\&T Partnership; the National Trans police Association; Mermaids; Scottish Transgender Alliance), regionally (e.g. TREC; Yorkshire MESMAC), or locally (e.g. Norfolk Trans Forum; FTM Nottingham) and projects/organisations with a remit around improving the lives of trans people (e.g. TransBareAll; GIRES; GENDYS) as well as those with more tangential links with the trans community such as professional networks (e.g. BPS Psychology of Sexualities Section) and LGBT-friendly organisations (e.g. The Metropolitan Community Church). Although we cannot be certain that our survey reached people not involved in these groups, it is likely that some participants found out about - and completed - the survey by finding out about it via members of these organisations.

The sample employed here comprised 660 participants, representing a range of gender identities (i.e. those with a clear and constant gender identity as male or female; those with a non-binary gender identity; and those with no gender identity) and at various stages of transition (see Table 1 for a breakdown of the sample by gender identity and stage of transition).

[insert Table 1 about here]

Other demographic information (e.g. age, ethnic background, etc) was also collected. However, because of the length of the survey, and the fact that these (generic) demographic questions comprised the final section, a number of participants $(\mathrm{N}=277)$ did not complete this section of the survey, so the complete profile of the sample is unknown. Of the 383 who did provide demographic information, $94.0 \%$ self-defined their ethnic background as 'white' (87.0\% English/Welsh/Scottish/Irish; 7.0\% from other white backgrounds) while just 5.4\% self-defined as from other ethnic backgrounds. The majority $83.3 \%$ resided in England, $11.5 \%$ in Scotland, $4.7 \%$ in Wales, with just $0.5 \%$ in other UK locations (i.e. Northern Ireland; British Crown Dependencies). Ages of these participants ranged from 18 to 78, with 
a mean age of 38 years. Although we cannot necessarily presume that the profile of the entire sample is approximated by these 383 participants, we can be fairly sure that the complete sample is overwhelmingly white and represents a wide range of ages.

The data for the entire survey was imported from Survey Monkey into SPSS where the statistical analyses were undertaken. For the purposes of this paper, the intent of which is to provide some baseline information, analyses comprise descriptive statistics about incidences of, and self-perceived risk of, transphobic victimisation. Where appropriate, analyses using Chi Square and Cramer's V tests were also undertaken to explore group associations by gender identity and by stage of transition. For the purposes of these analyses, the two non-binary gender identity categories (I have a constant and clear non-binary gender identity; I have a variable and fluid non-binary gender identity) and the category 'I have no gender identity' were collapsed into a single category titled 'non-binary'. Those self-identifying as 'unsure' of their gender identity were excluded from these analyses. Those self-identifying as 'unsure' about their stage of transition were excluded from analyses relating to stage of transition. .

\section{Results}

\section{Occurrence of transphobic victimisation}

This set of analyses pertains to questions asking about respondents' personal experiences of transphobic victimisation. While not exhaustive of the many forms that transphobic victimisation might take, the findings reported here pertain to three main types of transphobic incidents: Physical violence (i.e. being hit or beaten up; physical intimidation), sexual aggression (i.e. sexual assault; rape; sexual harassment; objectification or fetishisation) and social hostility (i.e. being made fun of; silent harassment; hearing that being trans is not normal). Table 2 presents a summary of the frequency with which respondents had experienced each of these types of incident.

[Insert table 2 about here]

Based on the responses to our survey, incidences of physical violence were relatively common. One in five respondents $(20.0 \%)$ reported having been hit or beaten up for being trans; $42 \%$ of whom ( $8.4 \%$ of all respondents) reported having been hit or beaten up a few 
times or many times. However, physical intimidation was much more frequently experienced with $39.5 \%$ of respondents reporting having been physically intimidated for being trans. Of these $63.2 \%$ ( $25.0 \%$ of all respondents) reported having been intimidated a few times or many times.

While some instances of rape and sexual assault were reported, these were the least commonly reported experiences of transphobic victimisation. Nevertheless, $6.9 \%$ of respondents reported having been raped and $14.7 \%$ having been sexually assaulted at least once because they are trans. Of those who reported being raped, $25.6 \%$ (1.8\% of all respondents) reported having been raped more than twice; $11.6 \%$ many times. The situation was reported to be similar for sexual assault with $31.5 \%$ (4.6\% of all respondents) reporting having been sexually assaulted for being trans - experiencing this a few times or many times. Acts of sexual intimidation were much more frequently reported with $40.3 \%$ of respondents reporting having experienced sexual harassment (e.g. cat calling; being propositioned) for being trans. Of these, $74.0 \%$ (29.8\% of all participants) had experienced this a few times or many times. Over half of respondents $(52.1 \%)$ reported having been objectified or fetishized sexually because they are trans; with $78.7 \%$ of those (41.0\% of all participants) having experienced this a few times or many times.

Social hostility comprised the most commonly reported transphobic incidents. Of all respondents, $75.0 \%$ reported having been made fun of or called names for being trans; an overwhelming majority, $80.9 \%$ of these ( $60.6 \%$ of all respondents), having experienced this a few or many times. Silent harassment - e.g. being stared at or whispered about - was even more common with $84.9 \%$ of respondents having experienced this. Of these, $93.3 \%(79.2 \%$ of all respondents) had experienced silent harassment a few or many times.

\section{$\underline{\text { Recency of transphobic victimisation }}$}

In order to ascertain the extent to which experiences of transphobic victimisation were current, we also asked participants how recently the various incidences they reported had happened. Many of the reported instances were surprisingly recent. For example, of those who reported being hit or beaten up $(\mathrm{N}=111) 12.6 \%$ had experienced this in the last year, while $24.3 \%$ of those who had faced physical intimidation or threats $(\mathrm{N}=181)$ had experienced this in the last year. Those experiencing sexual aggression in the last year was variable with $23.7 \%$ of those who had been raped $(\mathrm{N}=38), 19.2 \%$ of those who had been sexually assaulted (N=73), 36.0\% of those who had been sexually harassed $(\mathrm{N}=164)$, and 
$51.9 \%$ of those who had been objectified/fetishised ( $\mathrm{N}=212)$. Also, $51.9 \%$ of those who had experienced silent harassment $(\mathrm{N}=336)$ had experienced this in the past year. These statistics would seem to suggest that transphobic victimisation is still fairly prevalent.

\section{Group differences in victimisation}

Data on instances occurring in the past year were also analysed on the basis of gender identity and stage of transition to explore whether transphobic experiences were more prevalent for those with particular gender identities or at certain stages of transition. We chose to focus solely on incidences in the past year primarily because the wide age range of respondents may mean that for many participants instances of transphobic victimisation may have occurred when they identified differently to now, or were at a different stage of transition. While those with a clear and constant identity as a woman tended to report more instances of transphobic victimisation occurring in the past year than did those of other gender identities, no significant associations were found for most forms of transphobic victimisation surveyed. The only factor for which a significant association was found was for silent harassment. For this factor there was an association between having a clear and constant identity as a woman and having experienced silent harassment in the past year $\left(\chi^{2}=9.56, \mathrm{df}=2, \mathrm{p}<.008\right)$ but the effect size was small ( $\mathrm{V}=.158)$. This would seem to suggest that there is a tendency for those with a clear and constant identity as a woman to be more likely to experience transphobic victimisation than those with a clear and constant identity as a man or with a non-binary gender identity. For some types of transphobic victimisation (i.e. being hit or beaten up, sexual assault, and rape) the group sizes were too small to calculate group associations on the basis of stage of transition. However, significant group associations were found for stage of transition for four types of transphobic victimisation surveyed; all with large effect sizes. Those currently undergoing a process (or part of a process) of gender reassignment or transition were significantly more likely to have reported experiencing physical harassment $\left(\chi^{2}=35.19, \mathrm{df}=3, \mathrm{p}<.000 ; \mathrm{V}=.402\right)$, sexual harassment $\left(\chi^{2}=24.63, \mathrm{df}=3, \mathrm{p}<.000 ; \mathrm{V}=.342\right)$, sexual objectification or fetishisation $\left(\chi^{2}=28.64, \mathrm{df}=3, \mathrm{p}<.000 ; \mathrm{V}=.327\right)$, and silent harassment $\left(\chi^{2}=40.93, \mathrm{df}=3, \mathrm{p}<.000 ; \mathrm{V}=.321\right)$ than those not proposing to undergo, those proposing to undergo, and those who had already undergone a process (or part of a process) of gender reassignment or transition.

$\underline{\text { Perceived Risk of Transphobic Victimisation }}$ 
When asked 'do you worry about... happening in the future?' the respondents answering yes were much higher in many cases than the percentage who had actually experienced those forms of transphobic victimisation. Fear of physical violence was particularly high with around two thirds of respondents indicating that they worried about being hit or beaten up $(66.2 \%)$ or physically intimidated $(67.5 \%)$ in the future. Similarly, fear of social hostility was equally high with $62.8 \%$ worrying about being made fun of and $64.7 \%$ worrying about experiencing silent harassment in the future. Fear of sexual violence was a little less - but disproportionately high - with around half of respondents indicating that they worried about being raped (50.1\%), sexually assaulted (54.2\%), sexually harassed (49.6\%) or objectified/fetishized (48.7\%) in the future. This would seem to suggest that many trans people perceive themselves to be 'at risk' of future victimisation. Many participants also reported personally knowing people who had been the victims of transphobically motivated physical violence, sexual assault, or even been killed; a factor likely to compound their selfperceived risk of future victimisation.

\section{Group differences in perceived risk of victimisation}

As other work has shown (e.g Ellis, McNeil \& Bailey, 2014), trans people are acutely aware that a lack of congruity in their gender presentation may potentially make them subject to victimisation. It would seem reasonable therefore to assume that a trans person may feel particularly at risk if their gender identity or their stage of transition meant that their appearance did not conform to socially expected gender norms. We therefore undertook analyses to explore self-perceived risk of transphobic victimisation in relation to both gender identity and stage of transition.

No statistical association was found for any of the types of transphobic victimisation on the basis of gender identity. Conversely, there was a clear association between stage of transition and self-perceived risk of transphobic victimisation. In relation to physical violence, a significant association was found between stage of transition and worrying about being hit or beaten up $\left(\chi^{2}=41.43, \mathrm{df}=3, \mathrm{p}<.000\right)$ or being subject to physical intimidation or threats $\left(\chi^{2}=28.42, \mathrm{df}=3, \mathrm{p}<.000\right)$. The effect size for the former was large $(\mathrm{V}=.29)$ while a medium effect size was found for the latter $(\mathrm{V}=.24)$. A significant association was also found between stage of transition and worrying about being raped $\left(\chi^{2}=12.79, \mathrm{df}=3, \mathrm{p}=.005\right)$, sexually assaulted $\left(\chi^{2}=9.67, \mathrm{df}=3, \mathrm{p}=.02\right)$, being objectified or fetishized $\left(\chi^{2}=9.07, \mathrm{df}=3, \mathrm{p}=.03\right)$, and being sexually harassed $\left(\chi^{2}=14.82, \mathrm{df}=3, \mathrm{p}=.002\right)$. For rape, sexual assault and objectification 
the effect size was small ( $\mathrm{V}=.17, \mathrm{~V}=.14, \mathrm{~V}=.13$ respectively) while the effect size for sexual harassment was medium ( $\mathrm{V}=.18$ ). Significant associations were also found for social hostility: worrying about being made fun of or called names $\left(\chi^{2}=52.07, \mathrm{df}=3, \mathrm{p}<.000\right)$ with a

large effect size $(\mathrm{V}=.30)$; and worrying about being subjected to silent harassment $\left(\chi^{2}=33.07\right.$, $\mathrm{df}=3, \mathrm{p}<.000)$ with a medium effect size $(\mathrm{V}=.25)$. In all instances reported here, those currently undergoing a process (or part of a process) of gender reassignment or transition were significantly more likely to worry about risk of future victimisation than were those who were not planning to undergo, had not undergone or who had already undergone a process (or part of a process) of transition. This would seem to suggest that those in the process of transitioning felt more vulnerable and therefore worried more about the possibility of transphobic hostility.

\section{Discussion}

The purpose of this paper was to report on trans people's actual experiences and selfperceived risk of transphobic victimisation and to explore differences in experiences and selfperceived risk in relation to gender identity and stage of transition. The findings of the present study showed that although reported levels of specific types of incident were variable, transphobic victimisation was prevalent and many respondents worried about being victims of transphobic incidents in the future.

Of the types of incident surveyed, instances of social hostility (being made fun of or called names; silent harassment) were the most commonly experienced form of transphobic victimisation, with most respondents having reported being subjected to this type of victimisation at least once. This is consistent with the findings of Turner et al's (2009) study where comments and verbal abuse were the most widely reported types of transphobic incident. Incidents of physical violence and certain types of sexual aggression (sexual harassment; being objectified/fetishized) were also reasonably frequently reported. The least reported experiences were having been hit or beaten up, raped or sexually assaulted. Due to variation in the recording of these details in different studies it is difficult to make direct comparisons, but the reported incidence of these types of transphobic victimisation were broadly similar to those reported elsewhere (e.g. see Couch et al., 2007; Nemoto et al., 2011). However, they would appear to be considerably higher than for the general population (see 
British Crime Survey www.gov.uk). This would seem to suggest that trans people are at substantially greater risk of victimisation than are cisgender people.

The findings of this study also indicate that transphobic victimisation is a current (and ongoing) issue. With the exception of being hit or beaten up, where only $12.6 \%$ of participants reported having been hit or beaten up for being trans in the last year, around a quarter to half of respondents who had experienced each of the surveyed forms of transphobic victimisation had experienced these in the last year. Furthermore, consistent with other recent work (e.g. Boza \& Nicholson Perry, 2014) many respondents reported having experienced the same type of transphobic victimisation on multiple occasions. The findings also highlighted that in most instances, between half and two thirds of all respondents worried about being the victim of each of these types of transphobic victimisation in the future.

The fact that the numbers of respondents fearing future victimisation far outstrips reports of actual victimisation is symptomatic of systematic oppression. In a culture that privileges cisgender experiences and devalues trans identities/lives, isolated incidences of victimisation invoke a heightened sense of fear in members of the wider trans community thus creating a climate of fear. As highlighted by Combs (2010) news of bad experiences travels quickly within the community, which he says can feel smaller than it really is, so that accounts of those incidences - whether actually experienced or 'witnessed' from a distance - linger and become personalised. These fears are also perpetuated by media representations of trans people (see XXXX) which tend to sensationalise and scrutinise the everyday experiences of trans people and, in addition, which draw particular attention to extreme incidents such as suicides and transphobic murders. These 'stories' of victimisation are often internalised by trans people culminating in a sense of vulnerability. This study also sheds some light on which groups within the trans population might potentially be more vulnerable to both victimisation and minority stress. Limited evidence was found in this study for differences related to gender identity in relation to both experiences and self-perceived risk of transphobic victimisation. However, the analyses reported here do seem to suggest an association between actual experience and stage of transition. For physical harassment, sexual harassment, sexual objectification/fetishisation, and silent harassment those currently undergoing a process (or part of a process) of gender reassignment or transition ${ }^{2}$ were

\footnotetext{
${ }^{2}$ Whilst we recognise that referring to transition as a process does not necessarily reflect the experiences of all trans people, and that even the concept of transition is itself open for discussion, we have referred to it as a process herein to reflect the language that we used in questions that were asked of participants.
} 
significantly more likely to have experienced transphobic victimisation in the last year than those at any other stage of transition. Similarly, while no statistical association was found between gender identity and self-perceived risk of transphobic victimisation, a strong association was observed for stage of transition. Here also, those currently undergoing a process (or part of a process) of gender reassignment or transition were significantly more likely to worry about being a victim of transphobia in the future. As highlighted elsewhere (see Ellis et al., 2014) this self-perceived risk may in part be due to confidence. Those undergoing gender reassignment are more likely to be seen as visibly trans and therefore have a heightened awareness of how others might perceive them. Also, because their appearance may be more obviously different from social norms they may potentially be easier targets for actual victimisation. In addition, many of our respondents indicated that they had experienced transphobic victimisation on multiple occasions, as well as in different forms. While this was beyond the scope of this paper, repeated experiences of victimisation could also be a contributing factor to heightened awareness of perceived risk. Whatever the underlying factors, these findings raise questions about the need to offer enhanced support to trans people through gender reassignment and the transition process more generally. Although not reported in this paper, many respondents indicated in their written responses the importance of social support in both undergoing the transition process and also successfully withstanding potential retribution.

Given the well-established link between transphobic victimisation and psychological distress (e.g. see Bariola et al., 2015; Boza \& Nicholson Perry, 2014; Nemoto et al., 2011), both the prevalence of transphobic victimisation and the levels of self-perceived risk of victimisation reported in this paper are of concern. While ideally this needs addressing at a societal level, the immediate impact on trans people themselves - especially those who are in the process of transitioning - would seem to suggest that there is a pressing need for dedicated support services. As highlighted elsewhere (e.g. see Bariola et al., 2015) providing trans-specific support services is a key factor in building resilience; especially for those who have experienced transphobic victimisation. The levels of victimisation reported here are indicative of the extent of cis-gender-centricity prevalent in society. This, coupled with a very limited awareness of the nature of trans people's lived experiences among service providers, means that mainstream services are ill-equipped to appropriately and effectively support trans people who experienced transphobic victimisation; particularly during transition (e.g. see Whittle et al., 2009). 
While this study provides a valuable insight into a previously underexplored topic, it is not without its issues. For reasons already outlined, the study relied on a non-probability sample, which makes it difficult to determine the extent to which the findings can be generalised to the wider trans population. Although the sample size should ameliorate this somewhat, the incomplete sample profile adds to the complexity of this issue in that we cannot establish the extent to which individuals from traditionally underrepresented groups (e.g. minority ethnic groups) are included within the sample. Also, in researching transphobic victimisation, this study has been necessarily reductionist in assuming that transphobic victimisation unproblematically constitutes victimisation that is motivated by transphobia. While respondents were specifically asked to report on experiences that had occurred 'because they were trans (or had a trans history)' we cannot necessarily presume that these incidents were transphobically motivated per se. As Meyer (2012) has highlighted - and many respondents themselves commented on - it is not always easy to determine the motivation of such victimisation as being unequivocally transphobic, as any number of related or intersecting characteristics (e.g. gender, sexual orientation, etc) may be factors in the victimisation of trans people. Furthermore, this is potentially compounded by the fact that reported incidences may have occurred at a time when a person's gender identity/presentation was very different from what it is now.

Given that clear links between poor mental health and victimisation are well established, the levels of victimisation and self-perceived risk reported in this paper are a cause for concern. Exploring the relationship between psychological distress and transphobic victimisation was outside the remit of this paper; but should be a priority for future research. While some studies have begun to explore the relationship between victimisation and mental health in trans populations (e.g. Bariola et al., 2015; Boza \& Nicholson Perry, 2014; Nemoto et al., 2011), a more nuanced analysis of this relationship would facilitate a better understanding of psychological distress and resilience in those undergoing gender reassignment or transition processes is sorely needed.

\section{Conclusion}

In summary, the analysis presented in this paper has provided some insight into the extent to which transphobic victimisation is a very real issue for many trans people. While the vast majority of respondents had experienced some form of victimisation, many had experienced a 
range of forms of victimisation; often on multiple occasions. Respondents were also acutely aware of the prevalence of transphobic victimisation, many perceiving themselves to be at risk of victimisation in the future; especially those undergoing a process of transition. This is the first study to explore group differences (by gender identity and stage of transition) in experiences of transphobic victimisation and self-perceived risk of future victimisation. The analysis has highlighted that those undergoing a process (or part of a process) of gender reassignment or transition are potentially at greater risk of transphobic victimisation; and also perceive themselves to be especially vulnerable to victimisation. On this basis, it is hoped that greater, and more specialised, services will be devoted to providing focused support to this sub-group of the trans population.

\section{References}

Bailey, L., McNeil, J., \& Ellis, S. J. (in press). 'I worry that they'll pick on someone I care about': The impact of mainstream British media on trans people's mental health and wellbeing. In A. Dhoest, L. Szulc, \& B. Eeckhout (Eds.), LBTGQs, media and culture in Europe. London: Routledge.

Bariola, E., Lyons, A., Leonard, W., Pitts, M., Badcock, P., \& Couch, M. (2015).

Demographic and psychosocial factors associated with psychological distress and resilience among transgender individuals. American Journal of Public Health, 105 (10), 2108-2116.

Bauer, G., Hammond, R., Pyne, J., Redman, N., Scanlon, K., Travers, A. and Travers, R. (2009), "TransPULSE", available at: http://transpulseproject.ca/ (accessed 5 November 2014).

Bell, J. G. \& Perry, B. (2015). Outside looking in: The community impacts of anti-lesbian, gay, and bisexual hate crime. Journal of Homosexuality, 62 (1), 98-120.

Boza, C. \& Nicholson Perry, K. (2014). Gender-related victimization, perceived social support, and predictors of depression among transgender Australians. International Journal of Transgenderism, 15 (1), 35-52. Combs, R. (2010). Where gender and medicine meet: Transition experiences and the NHS.

Unpublished Doctoral Thesis, the University of Manchester, UK.

Couch, M. et al (2007). Tranznation: A report on the health and wellbeing of transgender people in Australia and New Zealand. ARCSHS. Available at 
http://www.latrobe.edu.au/arcshs/downloads/arcshs-research-

publications/Tranznation_Report.pdf

Cramer, R.J., Burks, A. C., Stroud, C. H., Bryson, C. N. \& Graham, J. (2015). A moderated mediation analysis of suicide proneness among lesbian, gay and bisexual community members. Journal of Social and Clinical Psychology, 34 (7), 622-641.

Ellis, S. J., McNeil, J., \& Bailey, L. (2014). Gender, stage of transition and situational avoidance: AUK study of trans people's experiences. Sexual and Relationship Therapy, 29 (3), 351-364.

Herek, G. M., Gogan, J. C. \& Gillis, J. R. (1999). Psychological sequelae of hate-crime victimisation among lesbian, gay and bisexual adults. Journal of Consulting and Clinical Psychology, 67 (6), 945-951.

Hill, D. B. \& Willoughby, B. L.B. (2005). The development and validation of the genderism and transphobia scale. Sex Roles, 53 (7-8), 531-544.

Kitzinger, C. (1996). Speaking of oppression: Psychology, politics, and the language of power. In E. D.Rothblum \& L. A. Bond (Eds.), Preventing heterosexism and homophobia (pp. 3-19). Thousand Oaks:Sage.

Lombardi, E. (2009). Varieties of transgender/transsexual lives and their relationship with transphobia. Journal of Homosexuality, 56 (8), 977-992.

Lombardi, E. L., Wilchins, R. A., Priesing, D. \& Malouf, D. (2002). Gender violence: Transgender experiences with violence and discrimination. Journal of Homosexuality, 42 (1), 89-101.

Maison, D. (1995). Do we say what we think? Polish Psychological Bulletin, 26, 175-187.

McConahay, J. Hardee, B. \& Batts, V. (1981). Has racism declined in America? It depends on who is asking and what is asked. The Journal of Conflict Resolution, 25 (4), 563 579.

McDermott, E., Roen, K., \& Scourfield, J. (2008). Avoiding shame: Young LGBT people, homophobia, and self-destructive behaviours. Culture, Health \& Sexuality, 10 (8), 815-829. 
McNeil, J., Bailey, L., Ellis, S., Morton, J., \& Regan, M. (2012). Trans mental health study 2012. Report for the Scottish Transgender Alliance. Edinburgh: Scottish Transgender Alliance.

Meyer, D. (2012). An intersectional analysis of lesbian, gay, bisexual and transgender (LGBT) people's evaluation of anti-queer violence. Gender \& Society, 26 (6), 849873.

Meyer, I. (1995). Minority stress and mental health in gay men. Journal of Health and Social Behavior, 36, 38-56.

Nagoshi, J. L., Adams, K. A., Terrell, H. K., Hill, E. D., Brzuzy, S. \& Nagoshi, C. T. (2008). Gender differences in correlates of homophobia and transphobia. Sex Roles, 59 (7-8), $521-531$.

Nemoto, T., Böedeker, B. \& Iwamoto, M. (2011). Social support, exposure to violence and transphobia, and correlates of depression among male-to-female transgender women with a history of sex work. American Journal of Public Health, 101 (10), 1980-1989.

Reed, B., Rhodes, S., Schofield, P. \& Wylie, K (2009). Gender Variance in the UK: Prevalence, Incidence, Growth and Geographic Distribution. Retrieved from Gender Identity Research in Education Society (GIRES) website http://www.gires.org.uk/assets/Medpro-Assets/GenderVarianceUK-report.pdf

Sugano, E., Nemoto, T. \& Operario, D. (2006). The impact of exposure to transphobia on HIV risk behavior in a sample of transgendered women of color in San Francisco. AIDS and Behavior, 10 (2), 217-224.

Tebbe, E. N. \& Moradi, B. (2012). Anti-transgender prejudice: A structural equation model of associated constructs. Journal of Counseling Psychology, 59 (2), 251-261.

Testa, R.J., Habarth, J., Peta, J., Bockting, W. \& Balsam, K. (2015). Development of the gender minority stress and resilience measure. Psychology of Sexual Orientation and Gender Diversity, 2 (1), 65-77.

Turner, L., Whittle, S. \& Combs, R. (2009). Transphobic hate crime in the European Union. Report commissioned by The Equalities Review. Available at http://www.pfc.org.uk/pdf/EngenderedPenalties.pdf 
Table 1: Breakdown of Participants by Gender Identity and Stage of Transition

\begin{tabular}{llc}
\hline & Response Category & $\begin{array}{c}\text { \% of participants } \\
\text { (N) }\end{array}$ \\
\hline Gender identity: & I have a constant and clear gender identity as a man & $26.4 \%(167)$ \\
& I have a constant and clear gender identity as a woman & $40.0 \%(253)$ \\
& I have a constant and clear non-binary gender identity & $9.0 \%(57)$ \\
& I have a variable or fluid non-binary gender identity & $15.7 \%(99)$ \\
& I have no gender identity & $2.8 \%(18)$ \\
& I am unsure of my gender identity & $6.0 \%(38)$ \\
\hline Stage of Transition: & $\begin{array}{l}\text { I have not undergone and do not propose undergoing } \\
\text { any part of a process of gender reassignment or } \\
\text { transition }\end{array}$ & $10.9 \%(70)$ \\
& $\begin{array}{l}\text { I am proposing undergoing a process (or part of a } \\
\text { process ) of gender reassignment or transition }\end{array}$ & $18.2 \%(117)$ \\
& $\begin{array}{l}\text { I am currently undergoing a process (or part of a } \\
\text { process) of gender reassignment or transition }\end{array}$ & $35.9 \%(231)$ \\
& $\begin{array}{l}\text { I have undergone a process (or part of a process) of } \\
\text { gender reassignment or transition } \\
\end{array}$ & $30.0 \%(193)$ \\
\hline
\end{tabular}


Table 2: Transphobic incidents

\begin{tabular}{|c|c|c|c|c|c|}
\hline How often have you... & $\begin{array}{c}\text { Many } \\
\text { times } \\
\% \\
\end{array}$ & $\begin{array}{c}\text { A few } \\
\text { times } \\
\% \\
\end{array}$ & $\begin{array}{c}\text { Twice } \\
\%\end{array}$ & $\begin{array}{c}\text { Once } \\
\%\end{array}$ & $\begin{array}{c}\text { Never } \\
\%\end{array}$ \\
\hline $\begin{array}{l}\text { Been hit or beaten up for being } \\
\text { trans }(N=641)\end{array}$ & $\begin{array}{c}2.5 \\
(\mathrm{~N}=16)\end{array}$ & $\begin{array}{c}5.9 \\
(N=38)\end{array}$ & $\begin{array}{c}3.6 \\
(N=23)\end{array}$ & $\begin{array}{c}8.0 \\
(N=51)\end{array}$ & $\begin{array}{c}80.0 \\
(N=513)\end{array}$ \\
\hline $\begin{array}{l}\text { Experienced physical } \\
\text { intimidation/threats for being trans } \\
(\mathrm{N}=632)\end{array}$ & $\begin{array}{c}7.3 \\
(\mathrm{~N}=46)\end{array}$ & $\begin{array}{c}17.7 \\
(\mathrm{~N}=112)\end{array}$ & $\begin{array}{c}5.0 \\
(N=32)\end{array}$ & $\begin{array}{c}9.5 \\
(\mathrm{~N}=60)\end{array}$ & $\begin{array}{c}60.5 \\
(N=382)\end{array}$ \\
\hline $\begin{array}{l}\text { Been raped because you are trans } \\
(\mathrm{N}=624)\end{array}$ & $\begin{array}{c}0.8 \\
(N=5)\end{array}$ & $\begin{array}{c}1.0 \\
(\mathrm{~N}=6)\end{array}$ & $\begin{array}{c}1.3 \\
(\mathrm{~N}=8)\end{array}$ & $\begin{array}{c}3.8 \\
(N=24)\end{array}$ & $\begin{array}{c}93.1 \\
(N=581)\end{array}$ \\
\hline $\begin{array}{l}\text { Been sexually assaulted because } \\
\text { you are trans }(N=625)\end{array}$ & $\begin{array}{c}0.6 \\
(N=4)\end{array}$ & $\begin{array}{c}4.0 \\
(\mathrm{~N}=25)\end{array}$ & $\begin{array}{c}2.9 \\
(N=18)\end{array}$ & $\begin{array}{c}7.2 \\
(N=45)\end{array}$ & $\begin{array}{c}85.3 \\
(\mathrm{~N}=533)\end{array}$ \\
\hline $\begin{array}{l}\text { Experienced sexual harassment } \\
\text { (e.g. cat calling, being } \\
\text { propositioned) because you are } \\
\text { trans }(\mathrm{N}=630)\end{array}$ & $\begin{array}{c}6.0 \\
(N=38)\end{array}$ & $\begin{array}{c}23.8 \\
(\mathrm{~N}=150)\end{array}$ & $\begin{array}{c}2.7 \\
(\mathrm{~N}=17)\end{array}$ & $\begin{array}{c}7.8 \\
(N=49)\end{array}$ & $\begin{array}{c}59.7 \\
(\mathrm{~N}=376)\end{array}$ \\
\hline $\begin{array}{l}\text { Been objectified or fetishised } \\
\text { sexually because you're trans } \\
(\mathrm{N}=638)\end{array}$ & $\begin{array}{c}15.0 \\
(\mathrm{~N}=96)\end{array}$ & $\begin{array}{c}26.0 \\
(\mathrm{~N}=166)\end{array}$ & $\begin{array}{c}2.2 \\
(\mathrm{~N}=14)\end{array}$ & $\begin{array}{c}8.9 \\
(N=57)\end{array}$ & $\begin{array}{c}47.8 \\
(\mathrm{~N}=305)\end{array}$ \\
\hline $\begin{array}{l}\text { Been made fun of or called names } \\
(\mathrm{N}=649)\end{array}$ & $\begin{array}{c}20.3 \\
(N=132)\end{array}$ & $\begin{array}{c}40.3 \\
(N=262)\end{array}$ & $\begin{array}{c}5.9 \\
(N=38)\end{array}$ & $\begin{array}{c}8.5 \\
(N=55)\end{array}$ & $\begin{array}{c}25.0 \\
(N=162)\end{array}$ \\
\hline $\begin{array}{l}\text { Experienced silent harassment (e.g. } \\
\text { being stared at/whispered about) } \\
\text { for being trans } \\
(\mathrm{N}=636)\end{array}$ & $\begin{array}{c}41.3 \\
(N=263)\end{array}$ & $\begin{array}{c}37.9 \\
(N=241)\end{array}$ & $\begin{array}{c}2.2 \\
(N=14)\end{array}$ & $\begin{array}{c}3.5 \\
(\mathrm{~N}=22)\end{array}$ & $\begin{array}{c}15.1 \\
(\mathrm{~N}=96)\end{array}$ \\
\hline
\end{tabular}

